\title{
Gute Schmerztherapie bedeutet patientenorientiertes Verhalten
}

\author{
Edmund A.M. Neugebauera ${ }^{\mathrm{a}}$ Maike de Wit ${ }^{\mathrm{b}}$ \\ a Institut für Forschung in der operativen Medizin (IFOM), Fakultät für Medizin, Lehrstuhl für chirurgische Forschung, \\ Campus Köln-Merheim, Private Universität Witten/Herdecke, Köln, \\ ${ }^{\mathrm{b}}$ Medizinische Klinik, Universitätsklinikum Hamburg-Eppendorf, Deutschland
}

Herr X oder Frau Y muss ein Krankenhaus aufsuchen. In 50-70\% der Fälle ist der Grund hierfür ein akut auftretender oder ein stärker gewordener chronischer Schmerz. Herr X oder Frau Y haben Angst es könnte sich etwas «Schlimmes» dahinter verbergen, die Krankenhausatmosphäre ist ihnen in der Regel fremd - sie sind unsicher. In der Aufnahme/Ambulanz erhalten sie eine PID-Nummer, sie mutieren zum Patienten und - auch abhängig von der Stärke der Schmerzen - verlieren ihre Autonomie.

Angst, Unsicherheit und der Verlust selbstbestimmten Handelns kennzeichnen diese Ausgangssituation. Der Patient nimmt dies nur deshalb in Kauf, weil er kompetente Hilfe vom Arzt und vom medizinischen Personal erwartet. Ihm ist es primär gleichgültig, wer ihm hilft - Hauptsache ihm wird geholfen. Bezogen auf eine kompetente Schmerztherapie heißt dies, dass ihm ein interdisziplinär abgestimmtes, wissenschaftlich abgesichertes und auf seine Person bezogenes Schmerztherapiekonzept zuteil wird.

Die Realität an deutschen Krankenhäusern in Hinblick auf die Akutschmerztherapie sieht leider immer noch anders aus. In einer im Jahr 2005 durchgeführten europäischen Umfrage an 1508 Kliniken in 7 Ländern belegt Deutschland einen schlechten Mittelplatz mit gravierenden Defiziten in der adäquaten Patienteninformation und dem Vorhalten von integrieren Schmerzmanagementkonzepten von der Patientenaufnahme bis zur Entlassung. In nur 14\% der Kliniken findet eine am internationalen Wissensstand orientierte Patienteninformation statt, schriftliche Schmerztherapiekonzepte werden adäquat nur in 9\% der Kliniken vorgehalten. Eine Ausbildung des medizinischen Personals (Ärzte/Pflegepersonal) findet nur in $16 \%$ der Kliniken statt. Die Konsequenz sind unzureichend und schlecht therapierte Patienten, was mit einem erheblichen Vertrauensverlust des Patienten in das Team, dem er sich anvertraut, einhergeht. Für den Patienten hat der Schmerz eine hohe Bedeutung - den Erfolg einer medizini- schen Maßnahme macht er, da er die an ihm vorgenommenen Diagnose- und Therapieschritte kaum beurteilen kann, zuallererst an der Beseitigung seiner Schmerzen fest. Angst und Unsicherheit verstärken das Schmerzempfinden. Die Schmerzen zu nehmen und dem Patienten damit ein Stück Autonomie zurückzugeben, müssen primäre Ziele jeder Schmerztherapie sein. Die neuen S3-Leitlinien zur Therapie akuter und posttraumatischer Schmerzen (www.awmf-online.de) enthalten hierzu ausführliche Empfehlungen.

Den Herausgebern von ChiruRgische Gastroenterologie INTERDISZIPLINÄR ist in besonderer Weise zu danken, dass sie das Thema Schmerztherapie zum Hauptthema eines ganzen Hefts gemacht haben. In 8 Beiträgen werden aktuelle am Stand der Wissenschaft ausgerichtete Konzepte zur Therapie akuter, chronischer und tumorbedingter Schmerzen dargestellt [1-8]. Kennzeichen aller Beiträge ist die Betonung eines interdisziplinären Vorgehens bei der Schmerztherapie - denn dies ist bislang in Deutschland ein Schwachpunkt. Sowohl in der Akutschmerztherapie als auch in der Tumorschmerztherapie sind wir auf Kooperationen angewiesen. Kooperation heißt aber auch, Verantwortlichkeiten klar zu regeln und schriftlich zu fixieren. Diese müssen allen, die an der Versorgung beteiligt sind, bekannt sein. Nur so erlebt der Patient ein kompetentes Team. Für den Bereich der Akutschmerztherpie ist dies über die «Initiative Schmerzfreie Klinik» (www.tuv. com/de/qualitaetsmanagement) bereits umgesetzt. Die Tumorschmerztherapie hat in den letzten 10 Jahren durch die Einrichtung von Tumorzentren und Palliativstationen sowie durch die Hospizbewegung einen erheblichen Aufschwung genommen.

Allen Autoren dieses Themenhefts möchten wir ausdrücklich für ihre kompetenten Beiträge danken. Wir verbinden mit diesem Heft die Hoffnung, dass viele dieser Konzepte Eingang in die am Patienten orientierte Versorgung finden.

\begin{tabular}{ll}
\hline KARGER & @ 2007 S. Karger GmbH, Freiburg \\
Fax +49 7614520714 & Accessible online at: \\
$\begin{array}{l}\text { E-mail Information@Karger.de } \\
\text { www.karger.com }\end{array}$ & www.karger.com/cga
\end{tabular}

Prof. Dr. Edmund A.M. Neugebauer

Institut für Forschung in der Operativen Medizin (IFOM)

Fakultät für Medizin, Lehrstuhl für Chirurgische Forschung, Private Universität Witten/Herdecke

Ostmerheimer Straße 200, 51109 Köln, Deutschland

Tel. +49 221989 57-0, Fax -30

E-mail ifom-neugebauer-sek@uni-wh.de 


\section{Literatur}

1 Hinrichs A, Schulz K, Järvinen I, Neugebauer EAM: Chronifizierung postoperativer Akutschmerzen. Chir Gastroenterol 2007;23:7-12.

2 Haase O, Schwenk W: Chirurgische Maßnahmen zur schmerzreduktion bei abdominalchirurgischen Eingriffen. Chir Gastroenterol 2007;23:13-18.

3 Shamiyeh A, Zehetner J: Prozedurenspezifische Schmerztherapie bei der Cholezystitis. Chir Gastroenterol 2007;23:19-22.
4 Beckurts TE: Schmerztherapie beim akuten Abdomen - Ist eine verzögerte Schmerztherapie noch zeitgemäß? Chir Gastroenterol 2007;23:23-29.

5 de Wit M: Therapie chronischer Tumorschmerzen. Chir Gastroenterol 2007;23:30-37.

6 Simanski C: Postoperative Nausea und Emesis (PONV) in der chirurgischen Gastroenterologie. Chir Gastroenterol 2007;23:38-44.
7 Lankenau G, Fliege M, Goetz AE, Krause T: Möglichkeiten und Grenzen der interventionellen Schmerztherapie bei Tumorpatienten. Chir Gastroenterol 2007;23:45-50.

8 Radbruch L, Elsner F: Schmerztherapie in der Palliativmedizin. Chir Gastroenterol 2007;23:51-59. 